\title{
Modeling Small Group Behaviors in Large Crowd Simulation
}

\author{
Seung In Park, Yong Cao, Francis Quek \\ Virginia Polytechnic Institute and State University*
}

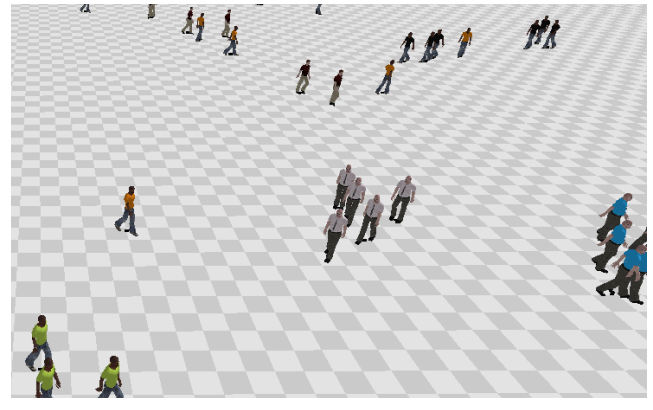

(a)

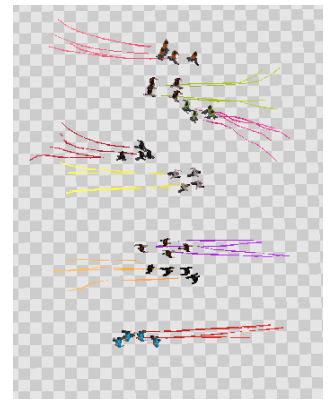

(b)

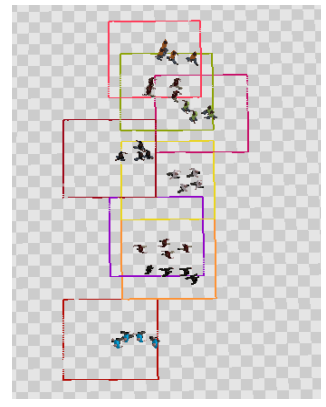

(c)

Figure 1: (a) A crowd of 1,156 groups is simulated in a large environment. Agents belonging to the same group stay and move together. (b) 8 groups move in opposite directions as forming lanes. (c) Each group's LIF is drawn.

\section{Introduction}

Large crowds are seldom made up solely of a mass of individuals. They typically also include large collections of small groups. However the most of existing approaches to crowd modeling treat a crowd either as a collection of isolated individuals, each maintaining its own goal, or as an aggregated entity in which large number of individuals share the same goal and behavior pattern.

Group members tend to move in a clustered way while avoiding collisions among themselves and with members of other groups. In addition, people respond only to the immediate surroundings but not to the entire world. It is only when they perceive direct evidence of a threat that they react. These observations suggest that the behavior of small groups may be modeled efficiently by having them to interact and react only within some locally defined space.

This paper presents a framework to simulate many number of small groups. To achieve this, we introduce a local interaction field(LIF) which embeds time-space information of surrounding environment of each group into the potential fields of a continuum dynamics simulation [Treuille et al. 2006].

\section{Our Approach}

At the initialization stage, each agent is assigned its group membership. A leader agent is randomly selected per group. Each group has its own initial and goal positions, and $\mathrm{A}^{*}$ path planning algorithm is performed per group. A hierarchical grid based approach is applied on this study, with the global path plan being executed only at the coarsest grid level. An example of a path between a start and goal location is shown by grey squares in Figure 2. For the reactive motion planning, the space opens up to a local grid within the corridor of a global path. We represent this as a LIF. This field is local since we have to be concerned only with the local navigation to the edge of the grid. Each group maintains its own LIF, and members of a group share a LIF. As a group traverses, the LIF is carried along with it to reflect the immediate surroundings. A LIF of a group and how it interacts with the global path grid are shown in Figure 2. We store a LIF as a 2D field of floating point numbers where the cell value represents the cost of the cell for the traversability. A high

*e-mail: (spark80, yongcao, quek)@vt.edu

Copyright is held by the author / owner(s)

I3D 2012, Costa Mesa, CA, March 9 - 11, 2012

ISBN 978-1-4503-1194-6/12/0003 \$10.00

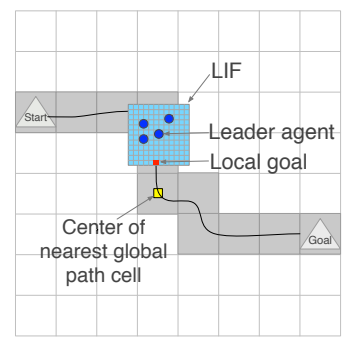

Figure 2: A global path on a coarse grid and LIF on a fine grid.

resolution grid is used for LIF to have a detailed control over agent movements.

The cost represents a measure of discomfort arising by the proximity of non-member agents and obstacles, and time-space factors including estimated travel time and length. A potential field to determine the optimal path within the LIF is computed using the cost. The potential field is assigned with zero value inside a goal, and the other grid cell values are approximated by solving the Eikonal equation. For further details on the potential field computation, see [Treuille et al. 2006]. Since we use a large scale of environment and the LIF covers only small portion of it, the goal position is likely to be outside the LIF. To resolve this problem, each group leader locates the local goal within a LIF for its group by intersecting the appropriate boundary edge of LIF and the projector line from the leader's position to the center of the nearest global grid cell that lies on the global path and is outside LIF.

The simulation results show that our model allows each small group to have own goal and emergent group movements. As shown in Figure 1, group members prefer to walk in tighter physical configurations, and form lanes when they encounter other groups. Currently we work on modeling of various type of group movements and improving social functionality of agents for group based movements.

\section{References}

Treuille, A., Cooper, S., And Popović, Z. 2006. Continuum crowds. ACM Trans. Graph. 25, 3, 1160-1168. 